\title{
Miscibility of sodium chloride and sodium dodecyl sulfate in the adsorbed film and aggregate
}

\author{
Hidemi Iyota $\cdot$ Rumen Krastev
}

Received: 17 October 2008 /Revised: 19 November 2008 / Accepted: 28 November 2008 / Published online: 23 January 2009

(C) Springer-Verlag 2009

\begin{abstract}
The adsorption, micelle formation, and salting out of sodium dodecyl sulfate in the presence of sodium chloride were studied from the viewpoint of their mixed adsorption and aggregate formation. The surface tension of aqueous solutions of a sodium chloride-sodium dodecyl sulfate mixture was measured as a function of the total molality and composition of the mixture. Phase diagrams of adsorption and aggregate formation were obtained by applying thermodynamic equations to the surface tension. Judging from the phase diagrams, sodium chloride and sodium dodecyl sulfate are miscible in the adsorbed film at very large composition of sodium chloride and in the saltedout crystalline particle, while they are immiscible in the micelle. The miscibilities in the adsorbed film, micelle, and crystalline particle increase in the following order: particle $>$ adsorbed film $>$ micelle. The difference in miscibility among the oriented states was ascribed to the difference in geometry between the adsorbed film and micelle and to the interaction between bilayer surfaces in the particle.
\end{abstract}

Keywords Mixture of sodium chloride and sodium dodecyl sulfate $\cdot$ Miscibility Adsorption and aggregate formation .

Micelle-particle equilibrium · Surface tension .

Thermodynamic treatment of surfactant mixture

\section{H. Iyota $(\bowtie)$}

Department of Life and Environmental Science,

Kagoshima Prefectural College,

Shimo-ishiki,

Kagoshima 890-0005, Japan

e-mail: iyota@k-kentan.ac.jp

R. Krastev

Max-Planck Institute of Colloids and Interfaces,

Golm 14476, Germany

\section{Introduction}

The adsorption and micelle formation of surfactants in the presence of inorganic salts have been studied from the viewpoint of additive effect of salts [1-5]. Enhanced adsorption and critical micelle concentration (CMC) lowering with added salts were observed for ionic surfactants. Tajima measured the adsorbed amounts of sodium dodecyl sulfate (SDS) in the presence of sodium chloride $(\mathrm{NaCl})$ by a direct radiotracer method and also evaluated the surface excesses of ions by an application of the Gibbs adsorption equation to the surface tension of aqueous $\mathrm{NaCl}$ solutions of SDS [6]. He found almost zero or slightly negative adsorption of counterions at the molarities of $\mathrm{NaCl}$ below $10 \mathrm{mM}$. Ozeki et al. evaluated the Gibbs surface excesses of surfactant ions, counterions, and coions from the surface tension of aqueous $\mathrm{NaCl}$ solutions of dodecyldimethylammonium chloride. They obtained small negative adsorption of counterions at low $\mathrm{NaCl}$ concentrations and a positive one at $\mathrm{NaCl}$ concentrations higher than $0.5 \mathrm{M}[4,7]$. In the above two studies, surface excesses of ions were evaluated from the dependence of surface tension on the respective concentrations of salt and surfactant.

However, thermodynamic treatment of surfactant mixture has been applied to mixtures of inorganic salt and surfactant in our previous studies [8-10]. We have measured the surface tension of aqueous solutions of a salt-surfactant mixture as a function of the total concentration and composition of the mixture instead of measuring it as a function of the respective concentrations of salt and surfactant. We then evaluated the composition of adsorbed film from the surface tension and obtained phase diagram of adsorption. The phase diagram was proved to be useful in clarifying the miscibility of inorganic salt and surfactant 
and the interaction between inorganic ions and the head group of surfactant in adsorbed films [8-13]. Furthermore, the adsorption and micelle formation have been studied separately in previous studies, even if the CMC was determined from the surface tension vs. concentration curve $[4,7,14,15]$. On the other hand, for salt-surfactant mixtures, we have determined the composition of the adsorbed film and micelle coexisting at the CMC from the dependence of mixture $\mathrm{CMC}$ and surface tension at the $\mathrm{CMC}$ on the bulk composition. We then compared the miscibilities of salt and surfactant in the adsorbed film and micelle by using the phase diagram for adsorbed filmmicelle equilibrium [8, 10-13].

It was found from the phase diagrams that (1) the polar head group of nonionic-surfactant molecule attract sodium ion a little in the adsorbed film and micelle, and (2) for a sodium chloride-dodecylammonium chloride mixture, dodecylammonium ions squeeze sodium ions out of the adsorbed film and micelle due to the electrostatic repulsion between the two cations [8-11]. As well as the miscibilities of sodium chloride with nonionic and cationic surfactants, it is of interest to investigate the miscibility of $\mathrm{NaCl}$ with an anionic surfactant in an adsorbed film and micelle.

Moreover, in the study of foam films, added salts cause the formation of black films stabilized with surfactants due to the reduction of electrostatic repulsion between the two surfaces in a film [16-18]. To clarify the formation of black films, it is essential for us to have knowledge of the miscibility and interaction of surfactant with salt in the surface of a film-forming solution and the changes in miscibility and interaction associated with the interaction between surfaces in a black film [17, 19-22].

Ionic surfactants are salted out at high salt concentrations, and hydrated crystalline particles (coagels) are formed in solutions [5, 23]. Nakayama and Shinoda [24] and Shinoda et al. [25] investigated the effect of added salts on the solubilities and Krafft points of ionic surfactants. At the points on the solubility curve above the Krafft point, singly dispersed surfactant is in equilibrium with hydrated solid surfactant and micellar surfactant. Hence, it is expected that useful information on adsorbed films, micelles, and/or salted-out particles can be obtained from the measurements of surface tension at high salt concentrations. However, the surface tension of aqueous solutions of ionic surfactants has hardly been measured at high salt concentrations except the studies by Ikeda et al. [7, 14, 2628]. Ikeda et al. ascribed the breaks on the surface tension vs. surfactant concentration curves even at high salt concentrations above $1 \mathrm{M}$ to micelle formation. On the other hand, Villeneuve et al. [29] succeeded in clarifying the vesicle formation and vesicle-micelle equilibrium of anionic and cationic surfactant mixture by surface tension. It is therefore of interest to examine how the salting out and equilibrium between micelles and crystalline particles can be elucidated by surface tension.

The aim of the present study is to clarify the miscibility and interaction of SDS with $\mathrm{NaCl}$ in the adsorbed film, micelle, and crystalline particle by applying thermodynamic treatment of surfactant mixture to a $\mathrm{NaCl}-\mathrm{SDS}$ mixture. The surface tension of aqueous solutions of the mixture was measured as a function of the total molality of the mixture and the mole fraction of SDS in the mixture over their wide ranges at $298.15 \mathrm{~K}$ under atmospheric pressure.

\section{Experimental}

Materials

SDS (Nacalai Tesque's guaranteed reagent) was purified by recrystallizing it triply from water and twice from ethanol. Its purity was checked by no minimum on the surface tension vs. concentration curve around the CMC. The purified SDS was kept under a dried atmosphere and reduced pressure in a desiccator to prevent its hydrolysis. Sodium chloride is the same sample as that used in the previous study [10]. It was stored under a dried atmosphere in a desiccator. Water, deionized and distilled, was further purified by distilling it twice from dilute alkaline solution of potassium permanganate.

\section{Measurements of surface tension}

Equilibrium surface tension was measured by the drop volume technique previously described [30], as a function of the total molality $\widehat{m}$ of $\mathrm{NaCl}$ and SDS and the mole fraction $\widehat{X}_{2}$ of SDS in the mixture. Taking into account the dissociation of $\mathrm{NaCl}$ and SDS into ions in solutions, $\widehat{m}$ and $\widehat{X}_{2}$ are defined by

$\widehat{m}=2 m_{1}+2 m_{2}$

and

$\widehat{X}_{2}=2 m_{2} / \widehat{m}=m_{2} /\left(m_{1}+m_{2}\right)$,

where $m_{1}$ is the molality of $\mathrm{NaCl}$ and $m_{2}$ the one of SDS. The density of aqueous $\mathrm{NaCl}$ solutions was used for the calculation of surface tension because the concentration of SDS is very low [31, 32]. A glass tip at which a solution drop is formed was weakly coated with dimethyl polysiloxane (Siliconize L-25, Fuji Systems, Japan) to give reproducible values of surface tension for anionic surfactants [33]. Temperature was kept at $298.15 \mathrm{~K}$ within $0.01 \mathrm{~K}$ by immersing the measuring cell in a water thermostat. The errors inherent in the measurement were less than $0.05 \mathrm{mN} \mathrm{m}^{-1}$. 
Determination of solubility

The aqueous solutions of a $\mathrm{NaCl}-\mathrm{SDS}$ mixture became turbid at large $\widehat{m}$ and small $\widehat{X}_{2}$. Figure 1 shows the surface tension $\gamma$ vs. total molality $\widehat{m}$ curves for two typical $\widehat{X}_{2}$ at which turbidity takes place. The $\gamma$ of transparent solutions decreases with increasing $\widehat{m}$; in sharp contrast, that of turbid solutions (solid circles) increases. Therefore, the lowest $\widehat{m}$ at which turbidity occurs was determined by a break on the $\gamma$ vs. $\widehat{m}$ curve. Another break is observed on the curve of the higher $\widehat{X}_{2}$ at $\widehat{m}$ below the lowest $\widehat{m}$, indicating the onset of micelle formation. Turbidity hence occurs in micellar solutions at higher $\widehat{X}_{2}$ and in monomeric solutions at lower $\widehat{X}_{2}$. The solutions at concentrations far above the lowest $\widehat{m}$ became turbid within $20 \mathrm{~min}$ during its standing at $298.15 \mathrm{~K}$ in the water thermostat after preparation. However, the solutions at concentrations around the lowest $\widehat{m}$ were allowed to stand in the thermostat for $12 \mathrm{~h}$ before measurement so that equilibrium is reached because the lowest $\widehat{m}$ did not change after the aging of $12 \mathrm{~h}$.

\section{Results and discussion}

Surface tension and aggregate formation

Figure 2a shows the surface tension vs. total molality curves at constant $\widehat{X}_{2}$ for higher $\widehat{X}_{2}$, and Fig. 2b shows those for lower $\widehat{X}_{2}$ including the results in Fig. 1. The $\gamma$ vs. $\widehat{m}$ curves of single $\mathrm{NaCl}$ and $\mathrm{SDS}$ are in good agreement with those in previous studies [34-36]. The $\gamma$ vs. $\widehat{m}$ curve of mixture changes its shape regularly with the change in $\widehat{X}_{2}$ and has one or two breaks indicating the onset of micelle formation and/or turbidity. The onset of turbidity, which is the formation of crystalline particle, is denoted by an arrow

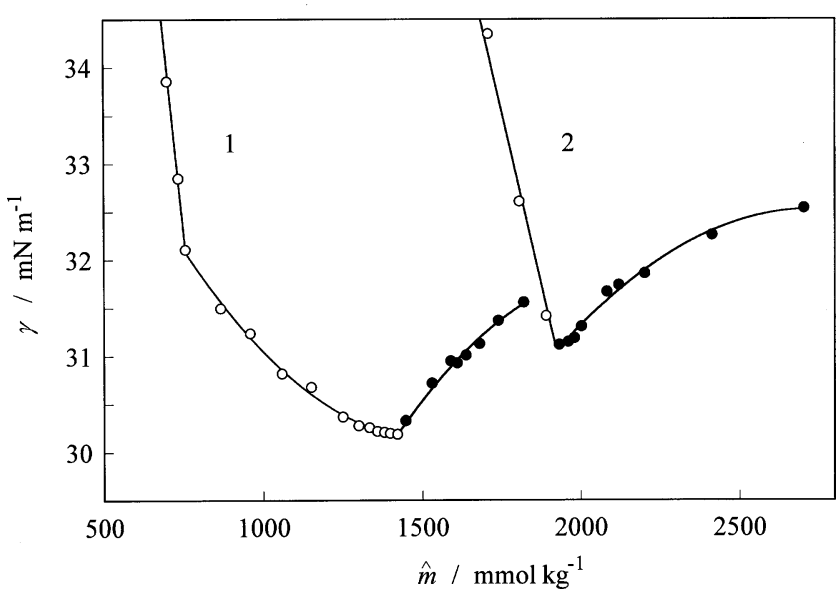

Fig. 1 Surface tension vs. total molality curves at constant composition: $\widehat{X}_{2}=(1) 0.00146$, (2) 0.000214; filled circle turbid solution

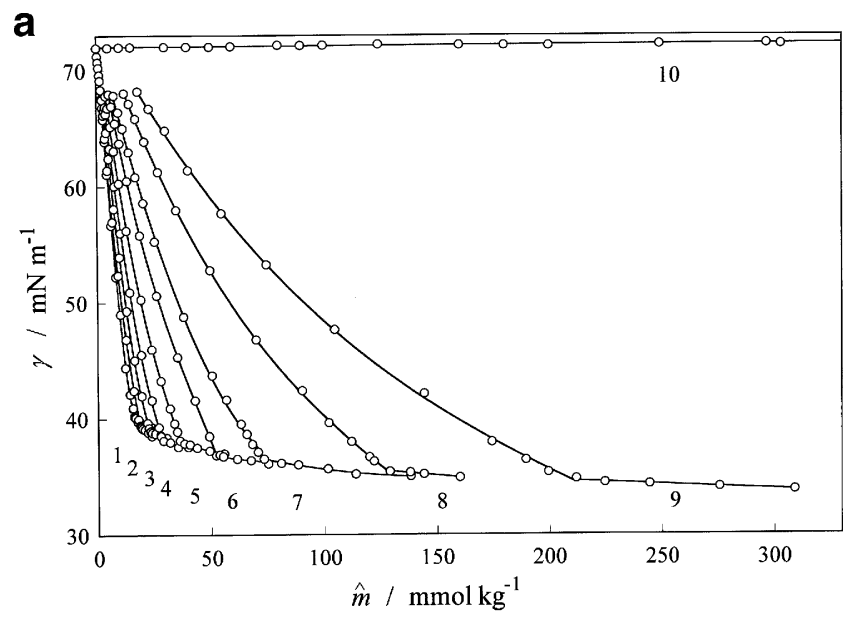

b

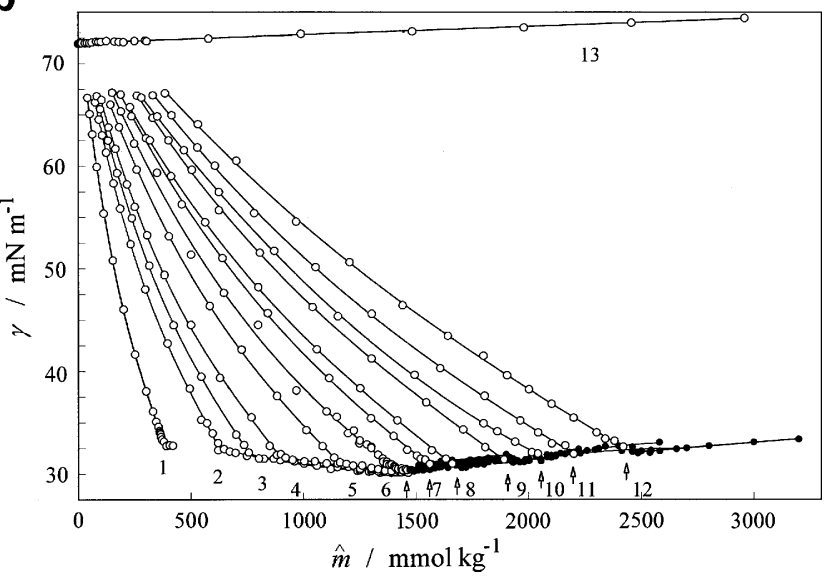

Fig. 2 Surface tension vs. total molality curves at constant composition: a $\widehat{X}_{2}=(1) 1,(2) 0.7893$, (3) 5,826 , (4) 0.3970, (5) 2,517, (6) 0.1303, (7) 0.0790, (8) 0.0315 , (9) 0.0135 , (10) 0 ; b $\widehat{X}_{2}=(1) 0.005012$, (2) 0.001997, (3) 0.001462, (4) 0.001050, (5) 0.0006592 , (6) 0.0004569 , (7) 0.0003699, (8) 0.0002894, (9) 0.0002136, (10) 0.0001582, (11) 0.0001257, (12) 0.0000992, (13) 0; filled circle turbid solution. Arrows show the breaks at the critical particle concentration

for each $\widehat{X}_{2}$ in Fig. 2b. The CMC $\widehat{C}$ of the mixture and the lowest $\widehat{m}$ to bring about turbidity, which we call critical particle concentration $\widehat{C}^{\mathrm{P}}$, were determined from the breaks in Fig. 2. The $\widehat{C}$ and $\widehat{C}^{\mathrm{P}}$ are plotted as a function of $\widehat{X}_{2}$ in Fig. 3. The magnification in Fig. 3 shows that the $\widehat{C}$ vs. $\widehat{X}_{2}$ and $\widehat{C}^{\mathrm{P}}$ vs. $\widehat{X}_{2}$ curves meet at $\widehat{X}_{2}=0.00042$. Micelles and particles coexist in equilibrium at the intersection; we denote the $\widehat{C}$ and $\widehat{X}_{2}$ at the intersection by $\widehat{C}^{\text {eq }}$ and $\widehat{X}_{2}^{\text {eq }}$, respectively. It is noticed that the $\widehat{C}^{\mathrm{P}}$ increases with decreasing $\widehat{X}_{2}$ below the $\widehat{X}_{2}^{\text {eq }}$; however, it is almost constant above the $\widehat{X}_{2}^{\text {eq }}$. We will later examine the coexistence of micelles and crystalline particles at the $\widehat{C}^{\mathrm{P}}$ for $\widehat{X}_{2}$ higher than the $\widehat{X}_{2}^{\text {eq }}$. The logarithm of CMC of SDS vs. logarithm of the counterion concentration obtained from the $\widehat{C}$ vs. $\widehat{X}_{2}$ curve, known as the Corrin-Harkins plot [1], gave a straight line below approximately $0.18 \mathrm{~mol} \mathrm{~kg}{ }^{-1}$ of the 
$\mathrm{NaCl}$ concentration. However, the $\widehat{C}$ vs. $\widehat{X}_{2}$ curve is more useful than the Corrin-Harkins plot because the former gives the composition of mixed micelle as shown later. The surface tension $\gamma^{\mathrm{C}}$ at the $\widehat{C}$ and $\gamma^{\mathrm{P}}$ at the $\widehat{C}^{P}$, taken from Fig. 2, are plotted against $\widehat{X}_{2}$ in Fig. 4. It is worth noting that $\gamma^{\mathrm{C}}$ decreases as $\widehat{X}_{2}$ decreases, in contrast, $\gamma^{\mathrm{P}}$ increases in the range of $\widehat{X}_{2}$ below the $\widehat{X}_{2}^{\text {eq }}$ and remains almost constant above the $\widehat{X}_{2}^{\text {eq }}$, as depicted in the magnification.

Miscibility in the adsorbed film

The mole fraction $\widehat{X}_{2}^{\mathrm{H}}$ of SDS in the adsorbed film is expressed by

$\widehat{X}_{2}^{\mathrm{H}}=\Gamma_{2}^{\mathrm{H}} /\left(\Gamma_{1}^{\mathrm{H}}+\Gamma_{2}^{\mathrm{H}}\right)$,

where the surface density $\Gamma_{1}^{\mathrm{H}}$ of $\mathrm{NaCl}$ is defined as that $\Gamma_{\mathrm{Cl}^{-}}^{\mathrm{H}}$ of chloride ion $\mathrm{Cl}^{-}$, and $\Gamma_{2}^{\mathrm{H}}$ of $\mathrm{SDS}$ as $\Gamma_{\mathrm{DS}}^{\mathrm{H}}$ of dodecyl sulfate ion $\mathrm{DS}^{-}$[37]. Numerical values of $\widehat{X}_{2}^{\mathrm{H}}$ were evaluated by applying

$\widehat{X}_{2}^{\mathrm{H}}=\widehat{X}_{2}-2\left(\widehat{X}_{1} \widehat{X}_{2} / \widehat{m}\right)\left(\partial \widehat{m} / \partial \widehat{X}_{2}\right)_{T, p, \gamma}\left(1+\gamma_{1 \pm, \widehat{m}}\right)$,

(refer to Appendix) to the $\widehat{m}$ vs. $\widehat{X}_{2}$ curves in Fig. 5. Here, the $\widehat{m}$ vs. $\widehat{X}_{2}$ curves were obtained by taking the $\widehat{m}$ values at

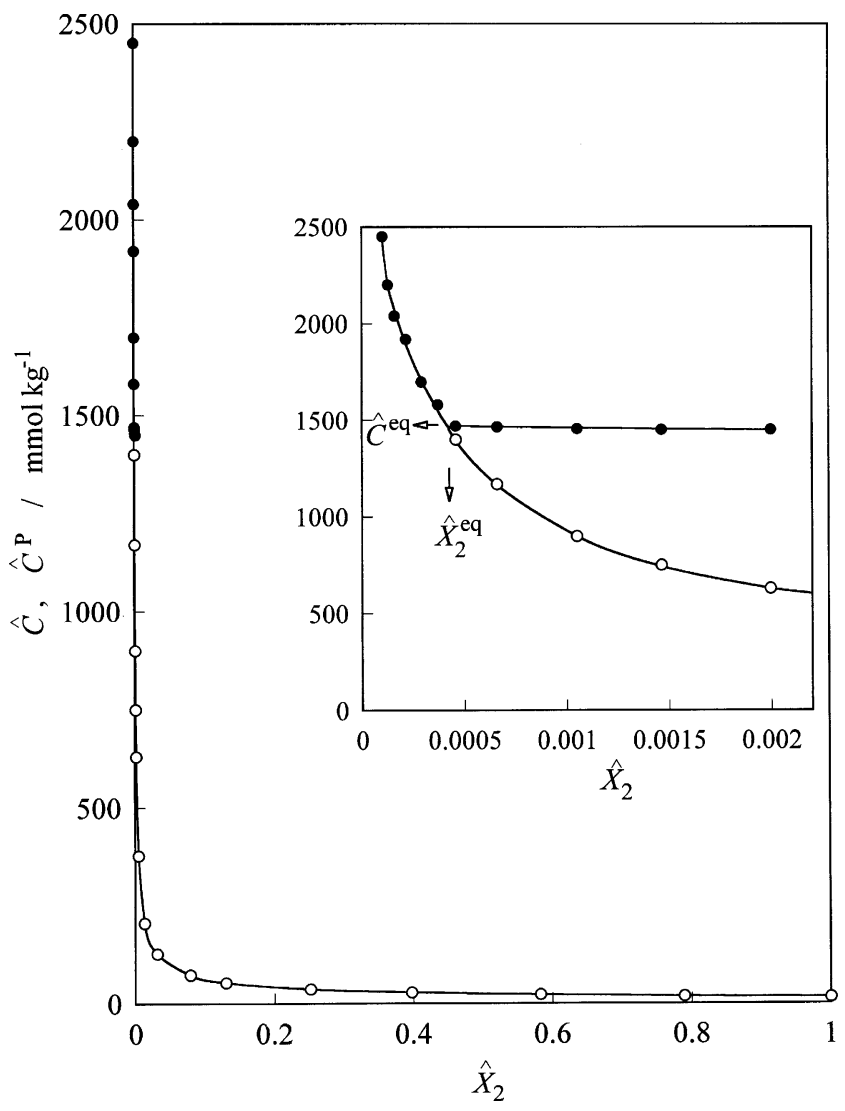

Fig. 3 Critical aggregate concentration vs. composition curves: empty circle $\widehat{C}$, filled circle $\widehat{C}^{\mathrm{P}}$

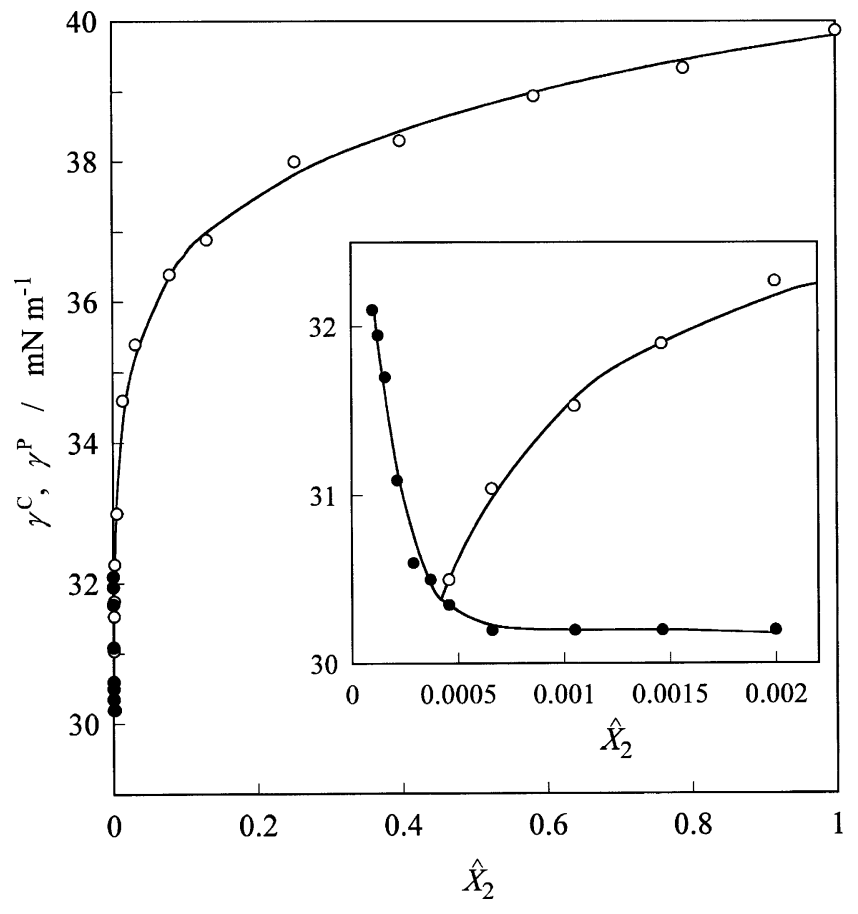

Fig. 4 Surface tension vs. composition curves at critical aggregate concentration: empty circle $\gamma^{\mathrm{C}}$, filled circle $\gamma^{\mathrm{P}}$

a given $\gamma$ from the $\gamma$ vs. $\widehat{m}$ curves in Fig. 2, and $\gamma_{1 \pm . \widehat{m}}$ is the dependence of mean activity coefficient $\gamma_{1+}$ of $\mathrm{NaCl}$ on $\widehat{m}$ defined by

$\gamma_{1 \pm, \widehat{m}}=\left(\partial \ln \gamma_{1 \pm} / \partial \ln \widehat{m}\right)_{T, p, \widehat{X}_{2}}$.

The literature value of $\gamma_{1 \pm}$ was used for the evaluation of $\widehat{X}_{2}^{\mathrm{H}}$ [38]. To draw the phase diagram of adsorption, the $\widehat{X}_{2}^{\mathrm{H}}$ values are shown in the form of $\widehat{m}$ vs. $\widehat{X}_{2}^{\mathrm{H}}$ curve together with the $\widehat{m}$ vs. $\widehat{X}_{2}$ curve, in Fig. 5 [37]. $\widehat{X}_{2}^{\mathrm{H}}$ is larger than unity at higher $\widehat{X}_{2}$, which shows the negative adsorption of $\mathrm{Cl}^{-}$by the definition of $\widehat{X}_{2}^{\mathrm{H}}$ (Eq. 3). Therefore, we conclude that the mixed adsorbed film of SDS with $\mathrm{NaCl}$ at higher $\widehat{X}_{2}$ is composed of SDS, and adsorbed $\mathrm{DS}^{-}$ions expel $\mathrm{Cl}^{-}$ions from the adsorbed film. Figure 5 further exhibits that the electrostatic repulsion between $\mathrm{DS}^{-}$and $\mathrm{Cl}^{-}$is enhanced at higher $\gamma$ because $\mathrm{DS}^{-}$is less densely packed in the adsorbed film at higher $\gamma$. Such repulsion of coions by surfactant ions is also observed for the adsorbed film of a mixture of $\mathrm{NaCl}$ and dodecylammonium chloride (DAC) [8].

It is noticed that $\widehat{X}_{2}^{\mathrm{H}}$ is smaller than unity at very small $\widehat{X}_{2}$. The $\widehat{X}_{2}^{\mathrm{H}}$ smaller than unity indicates that (1) $\mathrm{NaCl}$ and SDS are miscible in the adsorbed film, and (2) $\Gamma_{1}^{\mathrm{H}}$ is positive due to enhanced adsorption of $\mathrm{Na}^{+}$and highly weakened repulsion between $\mathrm{DS}^{-}$and $\mathrm{Cl}^{-}$in the adsorbed film. To show the miscibility in terms of surface density, we calculated the surface densities of individual ions by 


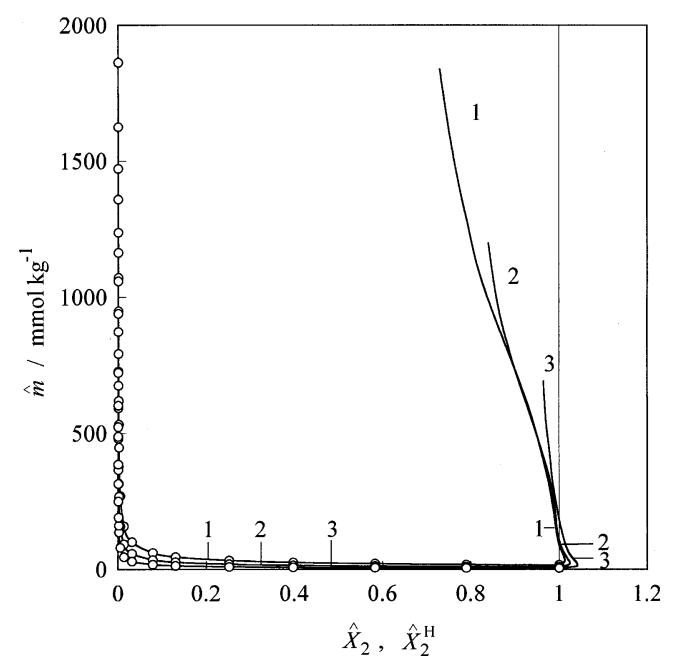

Fig. 5 Total molality vs. composition curves at constant surface tension: $\gamma=(1) 40,(2) 50$, (3) $60 \mathrm{mN} \mathrm{m}^{-1}$; empty circle $\widehat{m}$ vs. $\widehat{X}_{2}$, solid line $\widehat{m}$ vs. $\widehat{X}_{2}^{\mathrm{H}}$

substituting the values of $\widehat{X}_{2}^{\mathrm{H}}$ in Fig. 5 and the total surface density $\widehat{\Gamma}^{\mathrm{H}}$ into the following equations:

$\Gamma_{\mathrm{DS}^{-}}^{\mathrm{H}}=(1 / 2) \widehat{\Gamma}^{\mathrm{H}} \widehat{X}_{2}^{\mathrm{H}}$,

$\Gamma_{\mathrm{Cl}^{-}}^{\mathrm{H}}=(1 / 2) \widehat{\Gamma}^{\mathrm{H}}\left(1-\widehat{X}_{2}^{\mathrm{H}}\right)$,

and

$\Gamma_{\mathrm{Na}^{+}}^{\mathrm{H}}=(1 / 2) \widehat{\Gamma}^{\mathrm{H}}$,

where the surface density $\Gamma_{\mathrm{Na}^{+}}^{\mathrm{H}}$ of sodium ion $\mathrm{Na}^{+}$is given by

$\Gamma_{\mathrm{Na}^{+}}^{\mathrm{H}}=\Gamma_{\mathrm{Cl}^{-}}^{\mathrm{H}}+\Gamma_{\mathrm{DS}^{-}}^{\mathrm{H}}$

from electroneutrality condition and $\widehat{\Gamma}^{\mathrm{H}}$ was evaluated by applying Eq. 26 in Appendix to the $\gamma$ vs. $\widehat{m}$ curves in Fig. 1.

Figure 6 represents those surface densities as a function of $\widehat{X}_{2}$ at $40 \mathrm{mN} \mathrm{m}^{-1}$. The gradual increase in $\Gamma_{\mathrm{DS}^{-}}^{\mathrm{H}}$ with decreasing $\widehat{X}_{2}$ in the range of $\widehat{X}_{2}$ from 1 to approximately 0.002 is due to the electrostatic screening of the repulsion between the head groups of adsorbed $\mathrm{DS}^{-}$ions by the presence of adsorbed $\mathrm{Na}^{+}$. On the other hand, the negative $\Gamma_{\mathrm{Cl}^{-}}^{\mathrm{H}}$, the desorption of $\mathrm{Cl}^{-}$, in the range of $\widehat{X}_{2}$ from 1 to 0.04 shows the repulsion between $\mathrm{DS}^{-}$and $\mathrm{Cl}^{-}$in the adsorbed film. Furthermore, $\Gamma_{\mathrm{DS}^{-}}^{\mathrm{H}}=\Gamma_{\mathrm{Na}^{+}}^{\mathrm{H}}$ and $\Gamma_{\mathrm{Cl}^{-}}^{\mathrm{H}}=0$ at $\widehat{X}_{2}=0.04$, which shows adsorbed $\mathrm{DS}^{-}$is neutralized by $\mathrm{Na}^{+}$. Taking into consideration that $\mathrm{Na}^{+}$adsorbs at the surface of an adsorbed film of a nonionic surfactant caused by ion-dipole interaction between $\mathrm{Na}^{+}$and the polar head group of surfactant molecule $[9,11,12], \Gamma_{\mathrm{Na}^{+}}^{\mathrm{H}}$ larger than $\Gamma_{\mathrm{DS}^{-}}^{\mathrm{H}}$ at $\widehat{X}_{2}$ below 0.04 shows that $\mathrm{Na}^{+}$, after the neutralization, further adsorbs at the surface of the adsorbed film probably due to attractive interaction between $\mathrm{Na}^{+}$and the sulfinyl groups in the head group of adsorbed $\mathrm{DS}^{-}$[39]. Moreover,
Fig. 6 shows that the excess adsorption of $\mathrm{Na}^{+}$over the adsorption of $\mathrm{DS}^{-}$is accompanied by the positive adsorption of $\mathrm{Cl}^{-}$so as to satisfy the electroneutrality condition. Such positive adsorption of inorganic coions is also observed for the NaCl-dodecyldimethylammonium chloride system at large $\mathrm{NaCl}$ concentrations [7]. It is worth noticing that below $\widehat{X}_{2}=0.001, \quad \Gamma_{\mathrm{DS}^{-}}^{\mathrm{H}}$ decreases with decreasing $\widehat{X}_{2}$ whereas $\Gamma_{\mathrm{Na}^{+}}^{\mathrm{H}}$ and $\Gamma_{\mathrm{Cl}^{-}}^{\mathrm{H}}$ increase. The decrease in $\Gamma_{\mathrm{DS}}^{\mathrm{H}}$ is probably due to the penetration of $\mathrm{Na}^{+}$among the head groups of adsorbed $\mathrm{DS}^{-}$[40-42] and/or the repulsion between the head groups of neutralized $\mathrm{DS}^{-}$caused by the $\mathrm{Na}^{+}$accumulated around the head groups of $\mathrm{DS}^{-}$in the adsorbed film.

Miscibility in the aggregates

Miscibility of $\mathrm{NaCl}$ and SDS in the micelle can be examined by using the mole fraction $\widehat{X}_{2}^{\mathrm{M}}$ of SDS in the micelle defined by

$\widehat{X}_{2}^{\mathrm{M}}=N_{2}^{\mathrm{M}} /\left(N_{1}^{\mathrm{M}}+N_{2}^{\mathrm{M}}\right)$,

where $N_{1}^{\mathrm{M}}$ and $N_{2}^{\mathrm{M}}$ are respectively the excess numbers of $\mathrm{NaCl}$ and SDS in one micelle defined with respect to the spherical dividing surface around the micelle so as to make the excess number of water molecules zero [43]. The $\widehat{X}_{2}^{\mathrm{M}}$ was numerically evaluated by applying

$\begin{aligned} \widehat{X}_{2}^{\mathrm{M}}= & \widehat{X}_{2}-2\left(\widehat{X}_{1} \widehat{X}_{2} / \widehat{C}\right) \\ & \times\left(\partial \widehat{C} / \partial \widehat{X}_{2}\right)_{T, p}\left(1+\gamma_{1 \pm, \widehat{m}}\right),\end{aligned}$

(refer to Appendix) to the $\widehat{C}$ vs. $\widehat{X}_{2}$ curves in Fig. 3 .

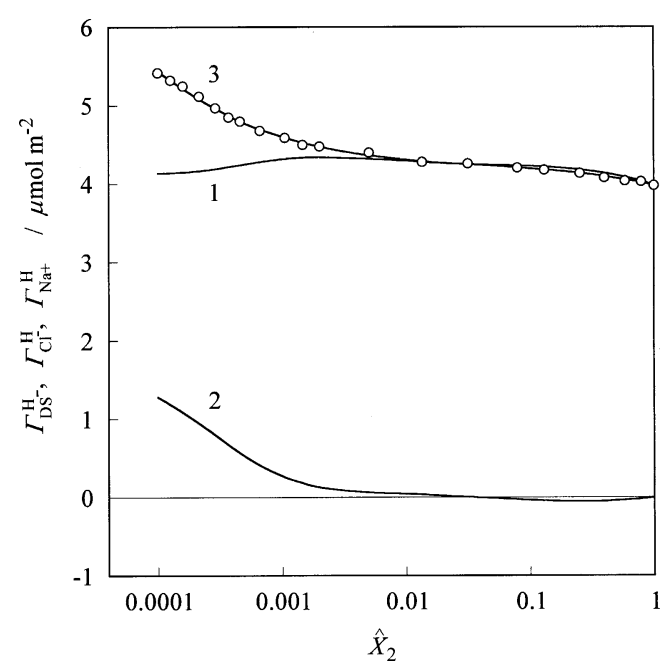

Fig. 6 Surface density vs. composition curves at $40 \mathrm{mN} \mathrm{m}^{-1}$ : (1) $\Gamma_{\mathrm{DS}^{-}}^{\mathrm{H}},(2) \Gamma_{\mathrm{Cl}^{-}}^{\mathrm{H}}$, (3) $\Gamma_{\mathrm{Na}^{+}}^{\mathrm{H}}$ 


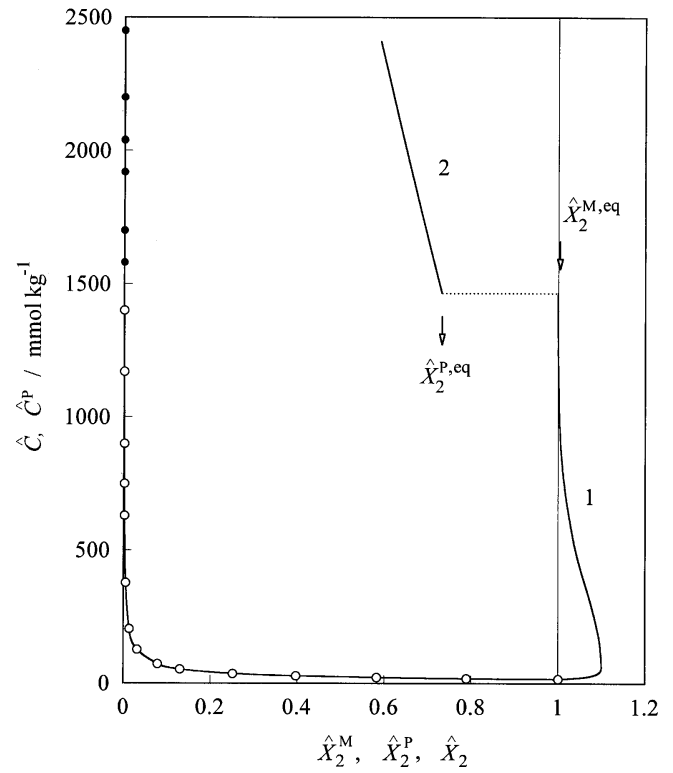

Fig. 7 Critical aggregate concentration vs. composition curves: (1) $\widehat{C}$ vs. $\widehat{X}_{2}^{\mathrm{M}},(2) \widehat{C}^{\mathrm{P}}$ vs. $\widehat{X}_{2}^{\mathrm{P}}$; empty circle $\widehat{C}$ vs. $\widehat{X}_{2}$, solid circle $\widehat{C}^{\mathrm{P}}$ vs. $\widehat{X}_{2}$

Since crystalline particles in solutions are coagels of the hydrated multilayers with a stacked bilayer structure in equilibrium with the surrounding solution $[44,45]$ and the particle concentration is extremely small at the $\widehat{C}^{\mathrm{P}}$, particle formation can be treated in a manner similar to micelle formation using the excess number $N_{1}^{\mathrm{P}}$ of $\mathrm{NaCl}$ and $N_{2}^{\mathrm{P}}$ of SDS in one particle defined with respect to the dividing surface, which makes the corresponding excess number of water molecules zero. The mole fraction $\widehat{X}_{2}^{\mathrm{P}}$ of SDS in the particle defined by

$\widehat{X}_{2}^{\mathrm{P}}=N_{2}^{\mathrm{P}} /\left(N_{1}^{\mathrm{P}}+N_{2}^{\mathrm{P}}\right)$

was calculated by applying

$$
\begin{aligned}
\widehat{X}_{2}^{\mathrm{P}}= & \widehat{X}_{2}-2\left(\widehat{X}_{1} \widehat{X}_{2} / \widehat{C}^{\mathrm{P}}\right) \\
& \times\left(\partial \widehat{C}^{\mathrm{P}} / \partial \widehat{X}_{2}\right)_{T, p}\left(1+\gamma_{1 \pm, \widehat{m}}\right),
\end{aligned}
$$

to the $\widehat{C}^{\mathrm{p}}$ vs. $\widehat{X}_{2}$ curve below $\widehat{X}_{2}^{\text {eq }}$ in Fig. 3 . We depict the resulting $\widehat{X}_{2}^{\mathrm{M}}$ and $\widehat{X}_{2}^{\mathrm{P}}$ in the form of $\widehat{C}$ vs. $\widehat{X}_{2}^{\mathrm{M}}$ and $\widehat{C}^{\mathrm{P}}$ vs. $\widehat{X}_{2}^{\mathrm{P}}$ curves, together with the $\widehat{C}$ vs. $\widehat{X}_{2}$ and $\widehat{C}^{\mathrm{P}}$ vs. $\widehat{X}_{2}$ curves, in Fig. 7. Figure 7 can be called phase diagram of aggregate formation because it shows the relations between the composition of aggregates and solution at the critical aggregate concentration $\widehat{C}$ or $\widehat{C}^{\mathrm{P}}$. The finding that $\widehat{X}_{2}^{\mathrm{M}}$ is larger than unity shows the expulsion of $\mathrm{Cl}^{-}$from the micelle by $\mathrm{DS}^{-}$. In contrast, $\widehat{X}_{2}^{\mathrm{P}}$ is smaller than unity and decreases with decreasing $\widehat{X}_{2}$. We therefore conclude that sodium ions highly adsorb at the bilayer surfaces in a particle, like at the surface of the adsorbed film at extremely small $\widehat{X}_{2}$.
Comparison between the miscibilities in the adsorbed film and aggregate

The miscibilities in the adsorbed film and aggregate can be compared with each other by use of the phase diagram for adsorbed film-aggregate equilibrium. We have

$\widehat{X}_{2}^{\mathrm{H}, \mathrm{C}}=\widehat{X}_{2}^{\mathrm{M}}-2\left(\widehat{X}_{1} \widehat{X}_{2} / R T \widehat{\Gamma}^{\mathrm{H}, \mathrm{C}}\right)\left(\partial \gamma^{\mathrm{C}} / \partial \widehat{X}_{2}\right)_{T, p}$

for the adsorbed film-micelle equilibrium and

$\widehat{X}_{2}^{\mathrm{H}, \mathrm{P}}=\widehat{X}_{2}^{\mathrm{P}}-2\left(\widehat{X}_{1} \widehat{X}_{2} / R T \widehat{\Gamma}^{\mathrm{H}, \mathrm{P}}\right)\left(\partial \gamma^{\mathrm{P}} / \partial \widehat{X}_{2}\right)_{T, p}$

for the adsorbed film-particle equilibrium (refer to Appendix), where $\widehat{X}_{2}^{\mathrm{H}, \mathrm{C}}$ and $\widehat{\Gamma}^{\mathrm{H}, \mathrm{C}}$ are the $\widehat{X}_{2}^{\mathrm{H}}$ and $\widehat{\Gamma}^{\mathrm{H}}$ at the $\widehat{C}$, respectively, and $\widehat{X}_{2}^{\mathrm{H}, \mathrm{P}}$ and $\widehat{\Gamma}^{\mathrm{H}, \mathrm{P}}$ are the corresponding ones at the $\widehat{C}^{\mathrm{P}}$.

The $\widehat{X}_{2}^{\mathrm{H}, \mathrm{C}}$ was calculated by applying Eq. 14 to the $\gamma^{\mathrm{C}}$ vs. $\widehat{X}_{2}$ curve in Fig. 4 and substituting the $\widehat{X}_{2}^{\mathrm{M}}$ values in Fig. 7 and the $\widehat{\Gamma}^{\mathrm{H}, \mathrm{C}}$ values into Eq. 14. In a manner similar to the evaluation of $\widehat{X}_{2}^{\mathrm{H}, \mathrm{C}}$, we obtained the numerical values of $\widehat{X}_{2}^{\mathrm{H}, \mathrm{P}}$ from the $\gamma^{\mathrm{P}}$ vs. $\widehat{X}_{2}$ curve. Figure 8 shows the $\widehat{X}_{2}^{\mathrm{H}, \mathrm{C}}$ and $\widehat{X}_{2}^{\mathrm{H}, \mathrm{P}}$ together with the $\widehat{X}_{2}^{\mathrm{M}}$ and $\widehat{X}_{2}^{\mathrm{P}}$ in the form of the surface tension vs. composition curves at the $\widehat{C}$ and $\widehat{C}^{\mathrm{P}}$. It is noticed that $\widehat{X}_{2}^{\mathrm{M}}>\widehat{X}_{2}^{\mathrm{H}, \mathrm{C}}$ and $\widehat{X}_{2}^{\mathrm{P}}<\widehat{X}_{2}^{\mathrm{H}, \mathrm{P}}$. The former inequality stems from the fact that the ionic head groups of $\mathrm{DS}^{-}$ions are less densely packed in the micelle than in the adsorbed film due to the difference in geometry between the adsorbed film and micelle. The latter one shows that the miscibility of $\mathrm{NaCl}$ and SDS in the adsorbed films of bilayers in a crystalline particle is larger than the

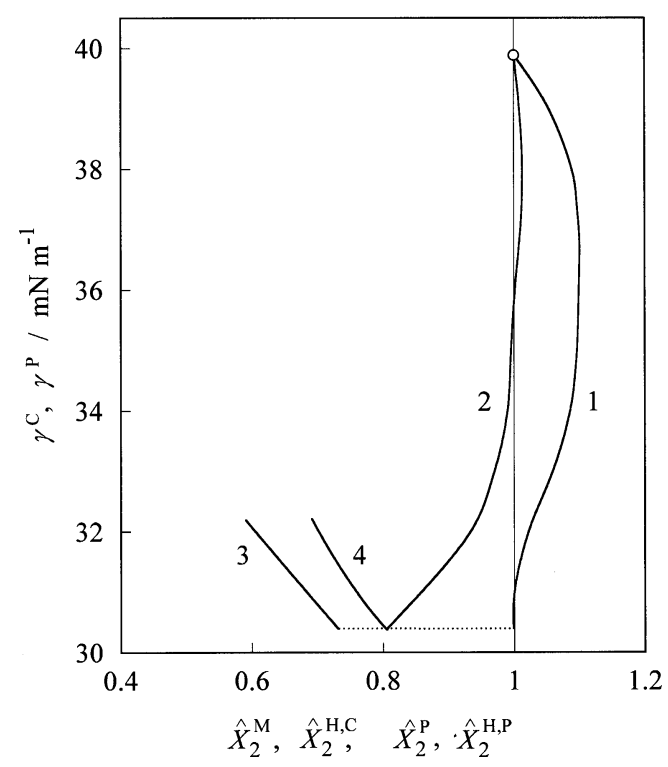

Fig. 8 Surface tension vs. composition curves at critical aggregate concentration: (1) $\gamma^{\mathrm{C}}$ vs. $\widehat{X}_{2}^{\mathrm{M}},(2) \gamma^{\mathrm{C}}$ vs. $\widehat{X}_{2}^{\mathrm{H}, \mathrm{C}},(3) \gamma^{\mathrm{P}}$ vs. $\widehat{X}_{2}^{\mathrm{P}},(4) \gamma^{\mathrm{P}}$ vs. $\widehat{X}_{2}^{\mathrm{H}, \mathrm{P}} ;$ empty circle $\gamma^{\mathrm{C}}$ of single SDS 
corresponding one in the adsorbed film at water/air interface. Taking into consideration that the curvature effect on miscibility is negligible for the adsorbed films of the bilayers because the crystalline particles are much larger than the micelles, we can attribute the $\widehat{X}_{2}^{\mathrm{P}}$ smaller than $\widehat{X}_{2}^{\mathrm{H}, \mathrm{P}}$ to larger adsorption of $\mathrm{Na}^{+}$at the bilayer surfaces compared with the corresponding one at the bulk surface. The above view is substantiated by the fact evidenced on the foam films stabilized with SDS and with decyl methyl sulfoxide that the adsorption of $\mathrm{Na}^{+}$at the surfaces of a Newton black film, a model of bilayer, is larger than the corresponding one of a thick film [17, 19-22].

The decrease in $\gamma$ of micellar solutions with increasing $\widehat{m}$ in Figs. 1 and 2 can be explained by comparing the $\gamma^{\mathrm{C}}$ vs. $\widehat{X}_{2}^{\mathrm{M}}$ curve in Fig. 8 with the $\gamma^{\mathrm{C}}$ vs. $\widehat{X}_{2}$ curve in Fig. 4 . The corresponding increase in $\gamma$ of turbid solutions with increasing $\widehat{m}$ at a given $\widehat{X}_{2}$ can be explained by comparing the $\gamma^{\mathrm{P}}$ vs. $\widehat{X}_{2}^{\mathrm{P}}$ curve with the $\gamma^{\mathrm{P}}$ vs. $\widehat{X}_{2}$ curve because the two curves form a phase diagram of particle formation: A transparent solution at a given $\widehat{X}_{2}$ becomes turbid, and particles appear at the $\widehat{C}^{\mathrm{P}}$ and further increase in the total molality at the $\widehat{X}_{2}$ causes an increase in the amount of particles and a decrease in the mole fraction of SDS in the solution surrounding the particles because the $\widehat{X}_{2}^{\mathrm{P}}$ is larger than the $\widehat{X}_{2}$ and the $\gamma^{\mathrm{P}}$ increases with decreasing $\widehat{X}_{2}$.

\section{Equilibrium between the micelle and crystalline particle}

Let us consider the micelle-particle equilibrium in solutions in the range of $\widehat{X}_{2} \geq \widehat{X}_{2}^{\text {eq }}$ and $\widehat{m} \geq \widehat{C}^{\text {eq }}$ shown in the magnification in Fig. 3. Mass balance relations are given by

$\widehat{m}=\widehat{C}^{\mathrm{eq}}+\widehat{m}^{\mathrm{M}}+\widehat{m}^{\mathrm{P}}$

for the total solutes and

$\widehat{m} \widehat{X}_{2}=\widehat{C}^{\mathrm{eq}} \widehat{X}_{2}^{\mathrm{eq}}+\widehat{m}^{\mathrm{M}} \widehat{X}_{2}^{\mathrm{M}, \mathrm{eq}}+\widehat{m}^{\mathrm{P}} \widehat{X}_{2}^{\mathrm{p}, \mathrm{eq}}$,

for SDS, where $\widehat{m}^{\mathrm{M}}$ and $\widehat{m}^{\mathrm{P}}$ are the $\widehat{m}$ ascribed to the micelle and particle, respectively, and $\widehat{X}_{2}^{\mathrm{M} \text {,eq }}$ and $\widehat{X}_{2}^{\mathrm{P}, \text { eq }}$ are the $\widehat{X}_{2}^{\mathrm{M}}$ and $\widehat{X}_{2}^{\mathrm{P}}$ at the $\widehat{X}_{2}^{\text {eq }}$ (see Fig. 7). As $\widehat{m}$ increases at a given $\widehat{X}_{2}$ above the $\widehat{X}_{2}^{\text {eq }}$ (see the magnification in Fig. 3), a micellar solution becomes turbid at the $\widehat{C}^{\mathrm{P}}$ where micelles are in equilibrium with an infinitesimal amount of crystalline particles, that is, $\widehat{m}^{\mathrm{P}} \approx 0$. Eliminating the $\widehat{m}^{\mathrm{M}}$ in Eqs. 16 and 17 and solving the resulting equation for $\widehat{m}$ at $\widehat{m}^{\mathrm{P}} \approx 0$, we have the relation between $\widehat{m}$ and $\widehat{X}_{2}$ for the micellar solutions at $\widehat{C}^{\mathrm{P}}$ :

$\widehat{m} \equiv \widehat{m}^{\mathrm{I}}=\widehat{C}^{\mathrm{eq}}\left(\widehat{X}_{2}^{\mathrm{eq}}-\widehat{X}_{2}^{\mathrm{M}, \mathrm{eq}}\right) /\left(\widehat{X}_{2}-\widehat{X}_{2}^{\mathrm{M}, \mathrm{eq}}\right)$.

Here $\widehat{m}^{\mathrm{I}}$ is defined as $\widehat{m}$ at which micelles coexist with an infinitesimal amount of particles at equilibrium. Equation 18 expresses the $\widehat{m}^{\mathrm{I}}$ vs. $\widehat{X}_{2}$ curve, one of the two micelle particle coexisting curves.
Further increase in $\widehat{m}$ at the $\widehat{X}_{2}$ causes the disappearance of micelles in the turbid solution at $\widehat{m}^{\mathrm{II}}$, the $\widehat{m}$ at which particles are in equilibrium with an infinitesimal amount of micelles, then we have $\widehat{m}^{\mathrm{M}}=0$. Eliminating the $\widehat{m}^{\mathrm{P}}$ in Eqs. 16 and 17 and solving the resulting equation for $\widehat{m}$ at $\widehat{m}^{\mathrm{M}}=0$ give

$\widehat{m}^{\mathrm{II}}=\widehat{C}^{\mathrm{eq}}\left(\widehat{X}_{2}^{\mathrm{eq}}-\widehat{X}_{2}^{\mathrm{P}, \mathrm{eq}}\right) /\left(\widehat{X}_{2}-\widehat{X}_{2}^{\mathrm{P}, \mathrm{eq}}\right)$

for the other coexisting curve $\widehat{m}^{\mathrm{II}}$ vs. $\widehat{X}_{2}$.

For the $\mathrm{NaCl}-\mathrm{SDS}$ mixture, we obtain $\widehat{C}^{\text {eq }}=$ $1,465 \mathrm{mmol} \mathrm{kg}^{-1}$ and $X_{2}^{\mathrm{eq}}=0.00042$ from Fig. 3 and $X_{2}^{\mathrm{M} \text {,eq }}=0.999$ and $\widehat{X}_{2}^{\mathrm{P}, \mathrm{eq}}=0.731$ from Fig. 7. Substitution of those values into Eqs. 18 and 19 leads to $\widehat{m}^{\mathrm{I}} \approx \widehat{m}^{\mathrm{II}} \approx \widehat{C}^{\text {eq }}$ for $\widehat{X}_{2}$ a little larger than the $\widehat{X}_{2}^{\text {eq }}$ because the $\widehat{X}_{2}^{\text {eq }}$ is much smaller than the $\widehat{X}_{2}^{\mathrm{M}, \mathrm{eq}}$ and $\widehat{X}_{2}^{\mathrm{P}, \mathrm{eq}}$. The experimental evidence shown in Fig. 3 that $\widehat{C}^{\mathrm{P}}$ is almost $\widehat{C}^{\text {eq }}$ in the range of $\widehat{X}_{2}$ above the $\widehat{X}_{2}^{\text {eq }}$ is consistent with the requirement $\widehat{m}^{\mathrm{I}} \approx \widehat{m}^{\mathrm{II}} \approx \widehat{C}^{\text {eq }}$ from Eqs. 18 and 19 . Since micelle formation can be regarded as an appearance of macroscopic phases [46, 47], the number of degrees of freedom for the solutions of the present mixture in which micelles and particles coexist at equilibrium can be approximated to be two from the Gibbs phase rule. The experimental evidence at $298.15 \mathrm{~K}$ and atmospheric pressure in Fig. 4 that $\gamma^{\mathrm{P}}$ is almost constant at $\widehat{X}_{2}$ above the $\widehat{X}_{2}^{\text {eq }}$ satisfies the requirement of phase rule.

In a future study, we will apply the present thermodynamic approach to a mixture of calcium chloride $\left(\mathrm{CaCl}_{2}\right)$ and SDS in which strong electrostatic attraction is expected between calcium ion and dodecyl sulfate ion in the adsorbed film and micelle, and the salting out will occur at around the bulk molar ratio $\mathrm{CaCl}_{2} / \mathrm{SDS}=1 / 2$.

\section{Conclusions}

The thermodynamic treatment of surfactant mixture has been applied to the NaCl-SDS mixture. The miscibility of $\mathrm{NaCl}$ and SDS in the adsorbed film was clarified by the use of the phase diagram of adsorption. Sodium chloride and SDS are immiscible in the adsorbed film at higher $\widehat{X}_{2}$ due to the electrostatic repulsion between adsorbed dodecyl sulfate ion and chloride ion. On the other hand, they are miscible in the adsorbed film at lower $\widehat{X}_{2}$ probably due to ion-dipole interaction between sodium ion and the head group of neutralized dodecyl sulfate ion.

The miscibility has been compared between the adsorbed film and micelle by the use of the phase diagrams of adsorption and micelle formation. Sodium chloride and SDS are immiscible in the micelle. The difference in miscibility between the adsorbed film and micelle can be ascribed to their difference in geometry. 
The salting out of SDS by $\mathrm{NaCl}$ has been clarified by measuring surface tension and treating the formation of crystalline particles in a manner similar to micelle formation. Judging from the phase diagram of aggregate formation, $\mathrm{NaCl}$ and SDS are miscible in the crystalline particle. The difference in miscibility between the adsorbed film and crystalline particle is attributable to the interaction between bilayer surfaces in the particle. The miscibilities in the adsorbed film, micelle, and particle coexisting at equilibrium were in the following order: particle $>$ adsorbed film $>$ micelle.

The equilibrium between micelles and particles in solutions has been thermodynamically considered by using the composition of micelle and particle at the $\widehat{X}_{2}^{\text {eq }}$. The coexisting curves $\widehat{m}^{\mathrm{I}} \approx \widehat{m}^{\mathrm{II}} \approx \widehat{C}^{\mathrm{eq}}$ in the range of $\widehat{X}_{2}$ a little larger than the $\widehat{X}_{2}^{\text {eq }}$ were in good agreement with the experimental results. The experimental evidence that the surface tension at the coexistence remained almost constant irrespective of $\widehat{X}_{2}$ was in conformity with the requirement of the Gibbs phase rule.

From the above, the application of thermodynamic treatment of surfactant mixture to the $\mathrm{NaCl}-\mathrm{SDS}$ mixture is confirmed to be useful.

\section{Appendix}

We apply the thermodynamic treatment of surfactant mixture $[37,43]$ to the NaCl-SDS mixture of which the aqueous solutions are nonideal [12]. The total differential of surface tension can be expressed for the mixture by

$$
\begin{gathered}
\mathrm{d} \gamma=-\Gamma_{\mathrm{Na}^{+}}^{\mathrm{H}} \mathrm{d} \widetilde{\mu}_{\mathrm{Na}^{+}}-\Gamma_{\mathrm{Cl}^{-}}^{\mathrm{H}} \mathrm{d} \widetilde{\mu}_{\mathrm{Cl}^{-}}-\Gamma_{\mathrm{DS}^{-}}^{\mathrm{H}} \mathrm{d} \widetilde{\mu}_{\mathrm{DS}^{-}} \\
=-\widehat{\Gamma}^{\mathrm{H}}\left(\widehat{X}_{1}^{\mathrm{H}} \mathrm{d} \mu_{1 \pm}+\widehat{X}_{2}^{\mathrm{H}} \mathrm{d} \mu_{2 \pm}\right)
\end{gathered}
$$

at constant temperature $T$ and pressure $p$, where the surface densities of ions are defined with respect to the two dividing planes chosen so as to make the excess numbers of moles of water and air zero [48], $\widetilde{\mu}_{j}$ is the electrochemical potential of $j$ ion, and $\mu_{i \pm}$ the mean chemical potential of component $i$. Here, the total surface density $\widehat{\Gamma}^{\mathrm{H}}$ is defined by

$\widehat{\Gamma}^{\mathrm{H}}=\Gamma_{\mathrm{Na}^{+}}^{\mathrm{H}}+\Gamma_{\mathrm{Cl}^{-}}^{\mathrm{H}}+\Gamma_{\mathrm{DS}^{-}}^{\mathrm{H}}=2\left(\Gamma_{1}^{\mathrm{H}}+\Gamma_{2}^{\mathrm{H}}\right)$

Since the molality $m_{\mathrm{Na}^{+}}$of the common ion $\mathrm{Na}^{+}$is given by

$m_{\mathrm{Na}^{+}}=m_{1}+m_{2}=\widehat{m} / 2$,

we have

$\mu_{i \pm}=\mu_{i \pm}^{\mathrm{o}}+R T \ln \left[\gamma_{i \pm}(\widehat{m} / 2)\left(\widehat{X}_{i}\right)^{1 / 2}\right],(i=1,2)$, where $\mu_{i \pm}^{\mathrm{o}}$ and $\gamma_{i \pm}$ are the mean standard chemical potential and mean activity coefficient of $i$, respectively. Substituting the total differential of $\mu_{i \pm}$ into Eq. 20 leads to

$$
\begin{aligned}
-\mathrm{d} \gamma & /\left(R T \widehat{\Gamma}^{\mathrm{H}}\right)=\left(1+\widehat{X}_{1}^{\mathrm{H}} \gamma_{1 \pm, \widehat{m}}+\widehat{X}_{2}^{\mathrm{H}} \gamma_{2 \pm, \widehat{m}}\right)(1 / \widehat{m}) \mathrm{d} \widehat{m} \\
& +\left[(1 / 2)\left(\widehat{X}_{2}^{\mathrm{H}}-\widehat{X}_{2}\right)+\widehat{X}_{1}\left(\widehat{X}_{1}^{\mathrm{H}} \gamma_{1 \pm, \widehat{X}_{2}}+\widehat{X}_{2}^{\mathrm{H}} \gamma_{2 \pm, \widehat{X}}\right)\right](24) \\
& \left(1 / \widehat{X}_{1} \widehat{X}_{2}\right) \mathrm{d} \widehat{X}_{2}
\end{aligned}
$$

where $\gamma_{i \pm, \widehat{X}_{2}}$ denotes

$\gamma_{i \pm, X_{2}}=\left(\partial \ln \gamma_{i \pm} / \partial \ln \widehat{X}_{2}\right)_{T, p, \widehat{m}},(i=1,2)$.

The ideal version of Eq. 24 was derived in the previous studies [37, 48]. We assume that $\gamma_{2 \pm, \widehat{m}} \approx \gamma_{1 \pm, \widehat{m}}$ and $\gamma_{i \pm, \widehat{X}_{2}} \approx 0$, considering that our measurements range in concentration from 0 to $2.5 \mathrm{~mol} \mathrm{~kg}^{-1}$ for $m_{1}$ and from 0 to approximately $10 \mathrm{mmol} \mathrm{kg}^{-1}$ for $m_{2}$ [7]. We then obtain from Eq. 24

$\widehat{\Gamma}^{\mathrm{H}}=-(\widehat{m} / R T)(\partial \gamma / \partial \widehat{m})_{T, p, \widehat{X}_{2}} /\left(1+\gamma_{1 \pm, \widehat{m}}\right)$

and

$$
\begin{aligned}
\widehat{X}_{2}^{\mathrm{H}}= & \widehat{X}_{2}-2\left(\widehat{X}_{1} \widehat{X}_{2} / \widehat{m}\right) \\
& \times\left(\partial \widehat{m} / \partial \widehat{X}_{2}\right)_{T, p, \gamma}\left(1+\gamma_{1 \pm, \widehat{m}}\right) .
\end{aligned}
$$

The equations for micelle formation corresponding to Eqs. 20 and 27 can be expressed by

$0=\left(\widehat{X}_{1}^{\mathrm{M}} \mathrm{d} \mu_{1 \pm}+\widehat{X}_{2}^{\mathrm{M}} \mathrm{d} \mu_{2 \pm}\right)$

and

$\widehat{X}_{2}^{\mathrm{M}}=\widehat{X}_{2}-2\left(\widehat{X}_{1} \widehat{X}_{2} / \widehat{C}\right)\left(\partial \widehat{C} / \partial \widehat{X}_{2}\right)_{T, p}\left(1+\gamma_{1 \pm, \widehat{m}}\right)$,

respectively. Equation 20 can be written as

$\mathrm{d} \gamma^{\mathrm{C}}=-\widehat{\Gamma}^{\mathrm{H}, \mathrm{C}}\left(\widehat{X}_{1}^{\mathrm{H}, \mathrm{C}} \mathrm{d} \mu_{1 \pm}+\widehat{X}_{2}^{\mathrm{H}, \mathrm{C}} \mathrm{d} \mu_{2 \pm}\right)$

at the $\widehat{C}$. Combination of Eqs. 28 and 30 and substitution of the total differentials of mean chemical potentials into the resulting equation give

$\widehat{X}_{2}^{\mathrm{H}, \mathrm{C}}=\widehat{X}_{2}^{\mathrm{M}}-2\left(\widehat{X}_{1} \widehat{X}_{2} / R T \widehat{\Gamma}^{\mathrm{H}, \mathrm{C}}\right)\left(\partial \gamma^{\mathrm{C}} / \partial \widehat{X}_{2}\right)_{T, p}$.

The equations for crystalline particle formation can be derived in a manner similar to micelle formation. 


\section{References}

1. Corrin ML, Harkins WM (1947) J Am Chem Soc 69:683

2. Mukerjee P (1965) J Phys Chem 69:4038

3. Ray A, Nemethy G (1971) J Am Chem Soc 93:6787

4. Ozeki S, Tsunoda M, Ikeda S (1978) J Colloid Interface Sci 64:28

5. Rosen MJ (1989) Surfactants and interfacial phenomena, 2nd edn. Wiley, New York, pp 137-138

6. Tajima K (1971) Bull Chem Soc Jpn 44:1767

7. Ozeki S, Ikeda S (1980) Bull Chem Soc Jpn 53:1832

8. Yamanaka M, Matsuki H, Ikeda N, Aratono M, Motomura K (1994) Langmuir 10:2950

9. Matsubara H, Ohta A, Kameda M, Villeneuve M, Ikeda N, Aratono M (1999) Langmuir 15:5496

10. Iyota H, Shimada K, Abe K, Ikeda N, Motomura K, Aratono M (2001) J Colloid Interface Sci 234:322

11. Iyota H, Tomimitsu T (2003) J Colloid Interface Sci 257:327

12. Iyota $\mathrm{H}$, Tomimitsu $\mathrm{T}$, Shimada $\mathrm{K}$, Ikeda $\mathrm{N}$, Motomura $\mathrm{K}$, Aratono M (2006) J Colloid Interface Sci 299:428

13. Iyota H, Tomimitsu T, Aratono M (2006) J Colloid Interface Sci 302:330

14. Okuda H, Ozeki S, Ikeda S (1984) Bull Chem Soc Jpn 57:1321

15. Johnson SB, Drummond CJ, Scales PJ, Nishimura S (1995) Langmuir 11:2367

16. Jones MN, Mysels KJ, Scholten PC (1966) Trans Faraday Soc 62:1336

17. de Feijter JA, Vrij A (1978) J Colloid Interface Sci 64:269

18. Exerowa D, Kolarov T, Khristov KHR (1987) Colloids Surf 22:171

19. Iyota H, Krustev R, Müller H-J (2004) Colloid Polym Sci 282:1329

20. Iyota H, Krustev R, Müller H-J (2004) Colloid Polym Sci 282:1392

21. Iyota H, Krustev R, Müller H-J (2005) Colloid Polym Sci 283:975

22. Iyota H, Krustev R, Müller H-J (2006) Colloid Polym Sci 284:455

23. Peacock JM, Matijević E (1980) J Colloid Interface Sci 77:548

24. Nakayama H, Shinoda K (1967) Bull Chem Soc Jpn 40:1797

25. Shinoda K, Yamaguchi N, Carlsson A (1989) J Phys Chem 93:7216

26. Okuda H, Ozeki S, Ikeda S (1987) J Colloid Interface Sci 115:155
27. Okuda H, Imae T, Ikeda S (1987) Colloids Surf 27:187

28. Tanaka A, Ikeda S (1991) Colloids Surf 56:217

29. Villeneuve M, Kaneshina S, Imae T, Aratono M (1999) Langmuir 15:2029

30. Motomura K, Iwanaga S, Hayami Y, Uryu S, Matuura R (1981) J Colloid Interface Sci 80:32

31. Chen C-TA, Chen JH, Millero FJ (1980) J Chem Eng Data 25:307

32. The Chem. Soc. Jpn (ed) (2004) Kagaku-binran, vol. 2, 5th rev. edn. Maruzen, Tokyo, p. II-9

33. Matsuki H, Aratono M, Kaneshina S, Motomura K (1997) J Colloid Interface Sci 191:120

34. The Chem. Soc. Jpn (ed) (1975) Kagaku-binran, vol. 2, 2nd rev. edn. Maruzen, Tokyo, p. 614

35. Mysels KJ (1986) Langmuir 2:423

36. Ikeda N, Matsuki H, Aratono M, Motomura K (1991) Rep Center Advan Instru Anal. Kyushu Univ 8:9

37. Motomura K, Aratono M (1993) In: Ogino K, Abe M (eds) Mixed surfactant systems. Marcel Dekker, New York, pp 99-144

38. Robinson RS, Stokes RH (2002) Electrolyte solutions, 2nd edn. Dover, New York, p 236

39. Laughlin RG (1994) The aqueous phase behavior of surfactants. Academic, New York, pp 246-248

40. Su TJ, Lu JR, Thomas RK, Penfold J (1993) J Phys Chem B 101:937

41. Warszyński P, Barzyk W, Lunkenheimer K, Fruhner H (1998) J Phys Chem B 102:10948

42. Warszyński P, Lunkenheimer K, Czichocki G (2002) Langmuir $18: 2506$

43. Motomura K, Yamanaka M, Aratono M (1984) Colloid Polym Sci 262:948

44. Clint JH (1992) Surfactant aggregation. Blackie, New York, pp 173-191

45. Laughlin RG (1994) The aqueous phase behavior of surfactants. Academic, New York, pp 181-237

46. Motomura K, Iwanaga S, Yamanaka M, Aratono M, Matuura R (1982) J Colloid Interface Sci 86:151

47. Motomura K, Iwanaga S, Uryu S, Matsukiyo H, Yamanaka M, Matuura R (1984) Colloids Sur 9:19

48. Motomura K, Ando N, Matsuki H, Aratono M (1990) J Colloid and Interface Sci 139:188 